Annuaire du Collège de France 2017-2018

\title{
Histoire turque et ottomane
}

\section{Edhem Eldem}

\section{OpenEdition}

\section{Journals}

Édition électronique

URL : https://journals.openedition.org/annuaire-cdf/15969

DOI : 10.4000/annuaire-cdf.15969

ISBN : 978-2-7226-0572-5

ISSN : 2109-9227

Éditeur

Collège de France

Édition imprimée

Date de publication : 30 décembre 2020

Pagination : $579-591$

ISBN : 978-2-7226-0516-9

ISSN : 0069-5580

Référence électronique

Edhem Eldem, « Histoire turque et ottomane », L'annuaire du Collège de France [En ligne], 118 | 2020,

mis en ligne le 01 avril 2021, consulté le 22 août 2022. URL : http://journals.openedition.org/annuairecdf/15969 ; DOI : https://doi.org/10.4000/annuaire-cdf.15969 


\title{
HISTOIRE TURQUE ET OTTOMANE
}

\author{
Edhem ELDEM \\ Professeur à l'université de Boğaziçi (Istanbul), \\ professeur invité au Collège de France
}

Mots-clés : Turquie, Empire ottoman, Occident

La série de cours « L'Empire ottoman et la Turquie face à l'Occident » est disponible, en audio et vidéo, sur le site internet du Collège de France (https://www.collegede-france.fr/site/edhem-eldem/course-2017-2018.htm), ainsi que le colloque «La découverte de la Phénicie au XIX siècle : Sidon entre la France, l'Empire ottoman et le Liban » (https://www.college-de-france.fr/site/edhem-eldem/p734167734517749_ content.htm). La leçon inaugurale disponible en audio et vidéo (https://www.collegede-france.fr/site/edhem-eldem/inaugural-lecture-2017-12-21-18h00.htm) a été publiée sous forme imprimée (Collège de France/Fayard) et numérique (Collège de France). Texte intégral en ligne : https://books.openedition.org/cdf/6192.

\section{ENSEIGNEMENT}

\section{COURS - L'EMPIRE OTTOMAN ET LA TURQUIE FACE À L'OCCIDENT}

\section{Introduction}

Le passé turc n'est pas qu'ottoman et l'histoire ottomane n'est pas que turque. C'est dire que l'intitulé de cette nouvelle chaire couvre un domaine très vaste et pour le moins complexe, voire ambigu. Nous porterons cependant notre regard sur un contexte plus ciblé qui, tout en réduisant l'ampleur du sujet, permettra d'intégrer ces deux dimensions de la question dans une réflexion historique particulière, celle de l'Empire ottoman et de la Turquie républicaine face à l'Occident. Ce questionnement s'inscrira à son tour dans une chronologie chevauchant les périodes moderne et contemporaine, du XVIII ${ }^{\mathrm{e}}$ siècle à nos jours.

Modernités, modernisation, occidentalisation, dynamiques internes, influences extérieures, cette période de transformations profondes est bien trop complexe pour se prêter à des lectures univoques qui finissent par donner une vision par trop simpliste, souvent faite d'une combinaison de triomphalisme occidental et de défaitisme ottoman. 
À un moment où l'histoire devient la proie d'une rhétorique politique des plus malsaines et où celle de l'Empire ottoman est soumise au lit de Procuste du nationalisme turco-islamique, il devient d'autant plus important de créer une plateforme capable de diffuser la connaissance historique dans ce domaine particulier et de promouvoir la recherche «en train de s'y faire », selon l'heureuse formule du Collège de France. C'est la mission que la chaire internationale d'histoire turque et ottomane s'est fixée pour les cinq années à venir, pendant lesquelles les spectaculaires transformations des trois derniers siècles seront examinées dans leurs dimensions politique, idéologique, sociale, économique et culturelle afin de saisir les dynamiques du changement et de la continuité dans l'une des régions les plus mouvementées du globe.

Le cours reprendra les grands moments de la période : intégration avec l'Europe au tournant du $\mathrm{XIX}^{\mathrm{e}}$ siècle, réformes étatiques des années 1820 et 1830 , rêves «ottomanistes» des années 1850 et 1860 , crise de 1876, autocratie hamidienne, révolution jeune-turque, débâcle de la Première Guerre mondiale... L'objectif premier est de combiner la synthèse et le détail et de familiariser le public avec l'étude critique de textes et de documents contemporains, ainsi qu'avec une approche diversifiée permettant de croiser des aspects différents mais convergents d'une réalité extrêmement variée. De la culture funéraire aux institutions financières, de l'anatomie des massacres aux biographies intellectuelles, de l'usage de la photographie à l'invention des ordres et décorations, de la naissance d'un orientalisme ottoman à la constitution d'une pratique archéologique, nombre d'études viendront se greffer sur ce récit central pour en dévoiler la richesse et la complexité.

\section{Cours 1 - Introduction}

\section{2 janvier 2018}

En introduction d'un programme portant sur une période longue - le fameux «long XIX ${ }^{\mathrm{e}}$ siècle »- et sur un sujet pour le moins complexe, il parait utile de consacrer le premier cours à des questions de fond qui concernent la discipline de l'histoire en général et, plus particulièrement, le domaine de l'histoire turque et ottomane. Revenant d'abord sur quelques-uns des problèmes posés lors de ma leçon inaugurale, j'ai commencé par rappeler à quel point le domaine était vicié par l'emprise du politique et de l'idéologique, notamment dans une Turquie que je qualifiais de «cliomane » et de « cliopathe».

Toutefois, c'est plutôt sur des problèmes inhérents à la discipline et relevant de questions de méthode et de contexte que je me suis penché, à commencer par la terminologie - particulièrement le risque d'amalgame, toutes périodes confondues, entre « turc » et «ottoman »- et par le monopole de fait que s'est arrogé la Turquie sur l'histoire ottomane, encouragée en cela par le rejet systématique par les autres États successeurs - balkaniques et arabes - de l'héritage ottoman, jugé incompatible avec leur propre récit «national». En rejetant un certain passé ottoman, ces traditions historiques créent de fait un « trou noir » historiographique, une parenthèse anhistorique qui réduit encore plus notre connaissance encore si incomplète de cet Empire, sans compter qu'il s'agit là d'un encouragement tacite à l'historiographie nationaliste turque de parfaire son emprise sur le sujet.

Évidemment, il fallait aussi évoquer l'impact de l'orientalisme, toujours très présent dans la discipline, notamment en raison de la survivance de certains réflexes eurocentriques des siècles précédents. Non sans ironie, le désir de corriger ces 
dérives historiographiques n'est pas dépourvu de risque, puisqu'il peut déboucher sur un anti-orientalisme qui devient parfois tout aussi réducteur et simpliste que sa cible. C'est l'un des défis majeurs de la discipline que de pouvoir restituer le droit à la parole à cet Orient - dont l'Empire ottoman - que l'orientalisme avait si longtemps défini comme objet et non comme sujet de l'histoire. Toutefois, cette restitution ne peut et ne doit se faire au prix de la rigueur historique, en empruntant des raccourcis intellectuels qui, évidemment pour la «bonne cause», s'attachent à inverser tout simplement l'argumentaire de l'érudition orientaliste. C'est trahir l'originalité et le génie d'Edward Said que de réduire le sens et l'impact de son Orientalism (1978) à une sorte de revanchisme tiers-mondiste censé rétablir un équilibre et une justice faussés par l'orientalisme. Naturellement, les exemples les plus crus de cette tendance sont facilement démasqués, mais il en va tout autrement d'une certaine production intellectuelle d'origine anglo-saxonne dont la subtilité et la sophistication garantissent le succès, et qui parvient souvent à créer l'illusion d'une véritable riposte historiographique. Or, il apparaît souvent que ces travaux reposent sur une rhétorique qui flatte certaines sensibilités idéologiques et morales et, surtout, sur le choix conscient d'une documentation partielle et partiale qui va dans le sens des conclusions souhaitées.

Cette dernière remarque m'amène au problème de la documentation, ou plutôt du document, dont l'usage en histoire ottomane reste encore extrêmement imparfait et problématique. Dans le camp « turc » le fétichisme documentaire domine, sacralisant le document à condition qu'il émane de l'État et, surtout, ignorant toute lecture critique, réduisant de fait le travail d'historien à un exercice de paléographie. Le problème, cependant, est plus général et touche aussi les cercles plus éclairés de la profession. L'inaccessibilité de certains fonds, la sous-exploitation de ceux qui sont accessibles, la tendance au «picorage» au détriment de traitements sériels et systématiques, les défis linguistique et paléographique découlant de la diversité culturelle de l'Empire... Autant de problèmes auxquels la discipline est confrontée et qui justifient que l'accent soit mis, tout au long du cours, sur des questions de méthode à partir de cas particuliers.

Enfin, pour un cours dont la grande ligne directrice reste chronologique, il convenait de rappeler que l'un des défis majeurs de l'historien travaillant sur cette période était de ne pas se laisser happer par une téléologie susceptible d'occulter les dynamiques internes de modernités locales qui sont soit ignorées, soit mises sur le compte d'une influence occidentale fort douteuse. S'il est évident que, par son intitulé, ce cours portera surtout sur une interaction avec l'Occident, il importe de se souvenir que les transformations de cette période ne peuvent et ne doivent pas être réduites à une logique aussi simpliste et univoque. Ainsi que je le rappelais à la fin de ma leçon inaugurale, l'objectif de l'historien, bien plus que de répondre à des questions, est d'en poser de bonnes, capables de révéler la complexité et les ambiguïtés du récit historique.

\section{Cours 2 - Curiosité et hésitations}

\section{9 janvier 2018}

Suite à l'introduction générale de la semaine précédente, cette première entrée en matière tente d'analyser la nature des premiers contacts entre les Ottomans et l'Europe, tout en soulignant qu'il ne s'agit pas à proprement parler d'une véritable découverte, puisque les Ottomans ont, depuis le début, été en contact avec un monde 
occidental d'abord italien, puis de plus en plus français. La véritable différence, au $\mathrm{XVIII}^{\mathrm{e}}$ siècle, tient à un changement sensible du rapport de forces entre les deux parties : tandis que les Ottomans, depuis la fin du XVII ${ }^{\mathrm{e}}$ siècle, commencent à perdre prise, leurs interlocuteurs occidentaux, eux, se font de plus en plus puissants et, souvent, arrogants. Il s'agit donc d'une situation nouvelle qui force les Ottomans à revoir leur politique envers un Occident de plus en plus envahissant et menaçant, mais aussi de plus en plus attrayant et tentant par ses succès.

Toutefois, avant de poursuivre cette interrogation sur les premiers contacts d'un genre nouveau entre les Ottomans et l'Occident, reprenant la promesse faite la semaine précédente d'aborder certains problèmes de méthode par le biais de cas particuliers, le cours emprunte un petit détour méthodologique autour du cas on ne peut plus particulier de «la couleur du cheval blanc de Franchet d'Espèrey ». En effet, toute la littérature historique, des années 1950 à nos jours, insiste sur la robe blanche de sa monture pour prouver que le général français, en investissant de la sorte la capitale ottomane en 1919, mettait en scène une riposte à la conquête de la ville par Mehmed II en 1453. Or des photographies contemporaines montrent bien que ce cheval n'était pas blanc, mais bai. Détail anodin, voire futile, certes, mais qui permet de se pencher sur deux questions primordiales en histoire. Tout d'abord, le problème de la manipulation des sources - généralement à des fins politiques ou idéologiques - et la faiblesse de la discipline face à cette désinformation, par manque de rigueur et de dépouillement systématique des sources. La deuxième interrogation, plus importante, porte sur le sens à donner à cette distorsion : loin d'être innocente, elle reflète une frustration ressentie par la population musulmane de la ville à la vue des forces d'occupation et, surtout, de l'enthousiasme des communautés non musulmanes. Ce n'est donc pas tant la correction d'une erreur factuelle qui compte, que la compréhension et la contextualisation des raisons qui la causèrent.

Retour au thème central du cours, le développement des relations entre Ottomans et Occidentaux pendant le XVIII ${ }^{\mathrm{e}}$ siècle, par un rappel de l'hospitalité « empoisonnée » et de l'attitude hautaine de la diplomatie ottomane, toujours unilatérale et subtilement dévalorisante, qui permet de comprendre la base de l'isolationnisme ottoman qui sera progressivement entamé par un désir et une obligation croissants d'ouverture vers l'Occident.

Encore une fois, il ne s'agit pas d'un revirement soudain qui ferait basculer la population ou l'élite dans une frénésie d'occidentalisation. Bien au-delà de la vision par trop simpliste d'un regard admiratif porté sur l'Occident - le "paradis des infidèles » - débouchant sur un désir d'émulation et d'inspiration trouvant son expression dans des éléments architecturaux ou des éléments décoratifs considérés comme précurseurs d'une occidentalisation à venir, il faut tenter de replacer ces innovations dans un contexte somme toute comparable à celui des turqueries en Europe. Il ne faut surtout pas oublier que la société ottomane est soumise à des dynamiques internes qui déclenchent des transformations et des changements que l'on peut aisément qualifier de « modernisation » sans pour autant les rattacher à une influence occidentale. Certains phénomènes socioculturels, comme l'évolution de l'épitaphe funéraire, montrent bien que cette société se transforme et se modernise sans pour autant avoir à s'inspirer d'un modèle occidental.

Il n'en est pas moins vrai que, dès les années 1720, on parvient à identifier une série d'événements qui, tout en restant superficiels, dénotent une curiosité croissante pour l'Europe : l'ambassade de Yirmisekiz Mehmed Çelebi (1720-1721) et celle de son fils Said Efendi (1741) ; les débuts timides de l'imprimerie du renégat İbrahim 
Müteferrika (1727-1742) ; les apports d'experts militaires occidentaux tels le comte de Bonneval (1730-1747) et le baron de Tott (1757-1763); les répercussions architecturales de l'adoption de formes baroques, comme dans le cas de la mosquée Nuruosmaniye (1748-1755); les premières représentations diplomatiques permanentes dans les principales capitales d'Europe; nombre de publications techniques, tel le Traité du sextant de Feyzi Bey (1805). Si un exemple se prête tout particulièrement à une lecture critique de la nature de cette nouvelle modernité, c'est bien le Tableau des nouveaux règlemens de l'Empire ottoman de Mahmud Raif Efendi (1798), publié au moment même où l'Empire se voyait confronté à un des événements les plus marquants et traumatisants de la période, l'expédition d'Égypte (1798), menée par un jeune général français du nom de Bonaparte.

\section{Cours 3 - Premiers signes d'engagement}

\section{6 janvier 2018}

L'expédition d'Égypte de Bonaparte (1798) est un tournant décisif dans l'histoire de l'Empire ottoman, mais aussi dans celle de la région tout entière et, surtout, des rapports entre Orient et Occident. Élément fondateur de l'orientalisme savant (Description de l'Égypte), coup d'envoi d'une politique coloniale française et britannique en Méditerranée et au Moyen-Orient, ébauche de la formation d'un État moderne en Égypte, cet événement constitue, pour les Ottomans, un traumatisme dont ils ne se remettront jamais entièrement. Incapable de répondre à ce coup de force par les armes, la Sublime Porte se retrouve à la merci d'alliances internationales pour assurer sa propre survie dans un environnement de plus en plus menaçant. La crise est résolue grâce au soutien britannique, mais, pendant toute la période napoléonienne, les Ottomans se retrouveront ballotés d'une alliance à une autre, navigant avec quelque succès sur les eaux de la diplomatie européenne. La puissance transformatrice de ce choc est énorme : la Porte sait désormais qu'elle ne peut plus se tenir à l'écart de la politique européenne et qu'elle est vraisemblablement vouée à un rôle secondaire et subordonné dans le nouvel ordre mondial.

Exemple flagrant de ces nouveaux enjeux diplomatiques, les Ottomans se voient obligés d'inventer un système de décorations - ordres et médailles - à l'occidentale afin de récompenser Nelson et ses braves. Jusqu'à la fin des années 1820, ces objets ne seront jamais utilisés pour récompenser les sujets de l'Empire. C'est là une caractéristique commune importante des nouveautés introduites: elles sont souvent «bonnes pour l'Occident», comme le Tableau des nouveaux réglemens de Mahmud Raif Efendi (1798), évoqué la semaine précédente, dont on n'a découvert que très récemment une version turque, jamais publiée.

Le problème est aggravé par le côté souvent formaliste des innovations. Le Nouvel Atlas (Cedid Atlas Tercümesi) du même Mahmud Raif (1803), traduction de l'ouvrage de William Faden (1793), en est un exemple parfait. En comparant la version ottomane de la carte des États-Unis à l'original de Faden, on observe un phénomène surprenant de traduction littérale de la toponymie, qui révèle une approche stérile d'un point de vue pratique, géographique et surtout maritime. Traduire « Cape Fear » par «Korku Burnu » revient à sacrifier l'utilité d'un outil de navigation à une précision linguistique tout à fait déplacée.

Au-delà du contenu problématique de ces outils de modernité, la véritable question qui se pose est celle de leur représentativité au sein de l'Empire. Sont-ils véritablement diffusés et reçus de manière assez significative, ou sommes-nous en 
train de «picorer » les plus beaux documents, laissant de côté une réalité bien moins glorieuse ? Alors que Mahmud Raif traduit l'atlas de Faden, l'historien Cabi Ömer Efendi révèle son ignorance profonde en relatant une version tout à fait fantaisiste de la création des colonies anglaises du Nouveau Monde. Comment l'érudition et l'ignorance coexistent-elles et, surtout, laquelle des deux est-elle plus représentative de la réalité ottomane?

Ce sont là des questions qui nous forcent à nous interroger sur la représentativité et l'impact de ces innovations. Des ouvrages ottomans traitent de l'Amérique depuis le début du $\mathrm{XVI}^{\mathrm{e}}$ siècle ; mais ce savoir reste, en surface, incapable de pénétrer les couches d'une population tenue à l'écart de cette production intellectuelle. D'où l'importance de ne pas se contenter d'examiner les documents (la production), mais d'essayer d'en étudier la diffusion (la distribution) et la réception (la consommation). C'est là une des faiblesses majeures de l'histoire ottomane, qui se limite souvent à prendre ces documents « au pied de la lettre », sans se soucier de l'ampleur ou même de l'absence d'une véritable réception. Il en découle aussi une prédilection pour les textes « canoniques » auxquels on attribue plus d'importance sans même savoir s'ils étaient vraiment lus.

Il faut aussi souligner une dernière faiblesse, soit la difficulté d'entendre de véritables « voix » qui, plutôt que les textes construits et conventionnels, rendraient possible une meilleure compréhension des mentalités. De tels textes sont rares, ne serait-ce que du fait de l'absence d'une tradition du procès-verbal comparable à celle de l'Occident. Ces voix sont quand même parfois audibles et identifiables, pour qui se donne la peine de les chercher, ainsi que le prouvent bien des hatt-ı hümayun (écrits impériaux), où le sultan s'exprime sans ambages et sans complexes. La note de Selim III à son vizir, dans laquelle il exprime sa satisfaction à la réception d'un portrait de Napoléon, en est un exemple fort intéressant qui sera étudié dans le détail lors du prochain cours.

\section{Cours 4 - Quand le doute s'installe}

\section{2 février 2018}

La semaine précédente, la discussion entamée autour de la note de Selim III à son vizir avait fourni l'occasion de se pencher sur la question des « voix » ottomanes et de la difficulté d'y accéder à travers une documentation assez pauvre en textes que l'on pourrait qualifier d' " ego-documents ». Une lecture au plus près de ce document dévoile la manière fort naïve dont le sultan se vantait auprès de son vizir d'en savoir plus que lui sur la culture et le savoir-vivre européens et se complaisait dans l'illusion que son échange de portraits avec Napoléon le mettait sur un pied d'égalité et d'intimité avec l'homme le plus puissant du globe. Ce sont là de précieux détails qui nous entrouvrent les portes de l'univers mental d'un Ottoman et que nous devons à la nature de ce document : une note presque verbale, rédigée à la hâte, sans sacrifier aux conventions d'un style épistolaire ou administratif.

Or, contrairement à leur forte présence en Europe occidentale, les mémoires et journaux ottomans sont extrêmement rares, de même que la correspondance entre les membres d'une faible minorité de gens maîtrisant la plume - dignitaires, bureaucrates, lettrés... Partout domine une documentation officielle, impersonnelle, variant du jargon administratif et fiscal de la gestion quotidienne de l'Empire au style ampoulé de la haute bureaucratie que seuls les kâtib ou scribes maîtrisent vraiment. Il n'est pas surprenant de voir que les sultans échappent jusqu'à un certain 
point à cette règle : ils ne sont guère soumis aux conventions et à l'étiquette, ce qui leur permet d'user d'un style beaucoup plus direct ; leurs « écrits impériaux » (hatt-l hümayun) ont force de décret et sont toujours rédigés de leur main ; et leur parole, comme leurs écrits, sont recueillis et conservés avec une attention toute particulière. C'est ainsi que l'on arrive plus facilement à « entendre » les pensées et les sentiments des sultans dans des missives amoureuses, au détour d'un décret, ou dans les écrits des chroniqueurs de la cour.

Évidemment, des exceptions sont là pour confirmer la règle et presque, dirait-on, pour accroître la frustration ressentie par l'absence ou l'extrême rareté de tels documents. En effet, certains textes révèlent des pensées ou un état d'esprit que la documentation officielle se garderait bien de laisser transparaître. C'est notamment le cas tout à fait fascinant d'une lettre de l'ambassadeur ottoman à la cour de Napoléon, Halet Efendi, qui met à nu toute une série d'inquiétudes face à un monde ressenti comme de plus en plus étranger et, surtout, de plus en plus menaçant. Partant de l'accusation de sodomie portée contre les musulmans par les Grecs et les Arméniens, perçus comme les suppôts de l'Occident, Halet Efendi défend sa « nation » en décrivant le Palais-Royal, haut lieu de la prostitution parisienne, pour conclure, soulagé, que les «Francs » sont bien pires en la matière. Au-delà de la confrontation de cet Ottoman avec un des clichés les plus tenaces de l'orientalisme et une morale hétéronormative qui lui est étrangère, le texte dévoile l'un des signes précurseurs d'une nouvelle phase dans l'occidentalisation : le doute. C'est lorsqu'ils commencent à douter de leur Empire, de leurs valeurs et d'eux-mêmes que les Ottomans musulmans - du moins les membres de l'élite - s'engagent de manière décisive dans la voie de l'occidentalisation, ou alors, dans sa condamnation systématique.

C'est ce doute et les hésitations qui s'ensuivent que l'on sent poindre dans l'œuvre de Şanizade Ataullah Efendi, dont l'Histoire (Tarih) a souvent et longtemps été vantée pour la «modernité » de son introduction (mukaddime). Fervent adepte de l'apport technique de la science occidentale - surtout la médecine et l'art de la guerre - dont il se fait le passeur par le biais de nombreuses traductions, Şanizade révèle aussi les limites de son engagement par un cas de plagiat qui vient grever son œuvre en tant que chroniqueur de la couronne. En effet, il s'avère que, dans sa célèbre introduction, Şanizade s'était «librement» inspiré de l'article «Histoire» de Voltaire dans l'Encyclopédie de Diderot et d'Alembert, mais qu'il avait aussi réussi à mettre son texte au goût du despote qu'était le sultan Mahmud II. Une étude détaillée de ce paradoxe révèle encore un aspect très particulier et fondamental de l'occidentalisation ottomane, soit l'adaptation opportuniste et peu scrupuleuse du canon européen dont on tend à conserver la forme sans vraiment se soucier du contenu.

\section{Cours 5 - Vers de nouveaux savoirs ?}

\section{9 février 2018}

J'avais annoncé la semaine précédente l'analyse que je ferais de la manière dont l'historien et chroniqueur Şanizade Ataullah Efendi, dont l'Histoire (Tarih) a souvent et longtemps été vantée pour la "modernité » de son introduction (mukaddime), s'était « librement » inspiré de l'article « Histoire » de Voltaire dans l'Encyclopédie de Diderot et d'Alembert. L'aspect le plus surprenant de la question était que Şanizade avait réussi à obtenir l'approbation et les éloges du sultan Mahmud II (r. 1808-1839) pour un texte émanant d'un auteur considéré, avec Rousseau, comme un mécréant 
(zındık) et un blasphémateur (kâfir). Évidemment, il n'y a pas vraiment de mystère : Şanizade s'était contenté d'adapter le texte de Voltaire afin de le rendre compatible avec l'idéologie conservatrice de l'establishment ottoman.

Une lecture plus détaillée du texte de Şanizade permet de mieux comprendre le modus operandi de cette adaptation. D'une manière générale, il apparaît que celui-ci avait procédé en usant de trois moyens principaux : omission, rajouts et distorsions. Des «statistiques » appliquées au texte en question montrent bien les dimensions de cette manipulation : le texte de Voltaire faisait environ 8500 mots ; celui de Şanizade n'en compte que la moitié (4 300), dont moins des deux tiers sont du philosophe (2600), le reste revenant à la créativité de Şanizade. Voltaire cite près d'une vingtaine d'historiens ; de ceux-ci, Şanizade ne retient qu'Hérodote, dont il écorche le nom en « Heredod».

Bien des omissions sont dues à l'ignorance et non à un choix, même politique ou idéologique : il est évident que Şanizade choisit de sauter et d'omettre bien des passages qui lui sont culturellement et intellectuellement inaccessibles. Ces lacunes et omissions sont évidentes lorsqu'il parle de l'histoire romaine, révélant son incapacité à distinguer les Grecs des Romains, qu'il appelle indifféremment Rûm. Plus surprenant, son traitement de l'histoire de l'Asie dévoile son ignorance de personnages comme Cyrus ou Oghuz Kagan qui devraient pourtant lui être familiers.

Les rajouts, au contraire, lui permettent de «corriger» Voltaire, notamment lorsque celui-ci ignore ou raille le fait religieux. C'est ainsi qu'il infuse une bonne dose de doctrine islamique dans son texte, renversant complètement la logique fondamentale du texte plagié. Enfin, des transpositions extrêmement brouillonnes et souvent déplacées visent à rendre les exemples «exotiques» de Voltaire compréhensibles au lecteur ottoman. Ainsi, les expéditions portugaises en Asie sont comparées à la reconquête du Hedjaz par les Ottomans à peine dix ans plus tôt, tandis que la découverte intellectuelle de la Chine par l'Europe est «traduite » par une longue digression sur la victoire de Murad Ir au Kosovo en 1389. Dans un cas comme dans l'autre, ces exercices d'adaptation se font l'occasion de chanter les louanges du sultan régnant et de ses ancêtres, contribuant à la « réussite » du chroniqueur auprès de son maître.

Ce cas très particulier met à nu certaines questions fondamentales, à commencer par la faiblesse de l'historiographie ottomane et turque qui a tout ignoré de cette généalogie textuelle pendant près de deux siècles. Plus encore, la « méthode » de Şanizade illustre certaines faiblesses intrinsèques de l'occidentalisation ottomane, notamment son désir de s'inspirer de l'Occident sans avoir à en assumer les implications intellectuelles et idéologiques. De toute évidence fasciné par le texte de Voltaire, mais ne pouvant ni ne voulant le plagier tel quel, Şanizade avait opté pour une demi-mesure qui en gardait la forme tout en en dénaturant le sens. Cet opportunisme utilitaire restera l'une des caractéristiques principales de l'occidentalisation ottomane et turque pendant les deux siècles à venir. Dans le cas particulier de ce plagiat, il est particulièrement désolant de voir que les successeurs de Şanizade n'y verront que du feu. Si le célèbre historien Cevdet Pacha - le Michelet ottoman - intègre mot pour mot la préface de Şanizade dans la sienne - tout en le citant, lui - c'est qu'il y voyait un grand mérite. Plus grave encore, jusqu'en 2015, aucun historien n'a même pensé à se poser la question de savoir d'où venaient ces étranges références aux marbres d'Arundel ou à Tlaxcala.

Question encore plus prenante, comment concilier ce fait avec la réalité incontestable de l'existence d'une historiographie ottomane qui, aux siècles précédents, a eu ses moments de gloire et qui, au XVII ${ }^{\mathrm{e}}$ siècle, peut être créditée de la 
redécouverte d'Ibn Khaldoun et de sa théorie de l'histoire? Car si Şanizade se décide à faire du mauvais Voltaire, cela veut dire qu'il sacrifie à ce caprice moderniste une accumulation de savoirs locaux. Ces savoirs et cette culture locaux qui, à force d'être délaissés, négligés, ou ignorés, finiront par s'effacer devant la montée en flèche de références et d'inspirations occidentales, souvent mal digérées.

\section{Cours 6 - Les défis du nouvel ordre}

\section{6 février 2018}

La référence à un «nouvel ordre » est double. Il s'agit avant tout d'un phénomène lié aux événements politiques qui suivirent la période napoléonienne, en particulier au congrès de Vienne (1815) dont l'objectif premier était de rétablir l'équilibre des puissances d'avant 1789 , dans le but avoué d'effacer et de prévenir les mouvements nationalistes, sécessionnistes ou unificateurs et tout soulèvement populaire jugé déstabilisant pour le système ainsi instauré. L'Empire ottoman, qui parvint à se tenir à l'écart des principaux conflits napoléoniens, ne participa pas au congrès, mais en fut l'un des objets, notamment par la question de la défense de son intégrité territoriale contre les mouvements indépendantistes et sécessionnistes des Balkans.

Pourtant, la rébellion grecque de 1821, qui prit bientôt des proportions de guerre d'indépendance, révéla le caractère éphémère de ces résolutions. Après des années de neutralité plus ou moins bienveillante, avec la bataille de Navarin (1827), les puissances européennes finirent par intervenir en faveur des insurgés, constituant ainsi la première entorse au système par la création d'une Grèce indépendante.

La notion de «nouvel ordre » est aussi un rappel du terme utilisé par Selim III pour décrire son programme de réforme fiscale et militaire, le Nizam-ı Cedid. Les Nouveaux règlemens de l'Empire ottoman de Mahmud Raif Efendi (1798) en étaient un excellent exemple. Toutefois, ce «nouvel ordre» fut aussi la cause d'une réaction menée notamment par les janissaires qui, se sentant menacés par le projet d'une nouvelle armée, s'insurgèrent et obtinrent la destitution de Selim III en 1807 et l'assassinèrent l'année suivante, lors de la contrerévolution qui mit le jeune Mahmud II sur le trône.

On comprend dès lors que, malgré des velléités de poursuivre l'œuvre de son cousin Selim III, Mahmud ait évité la moindre référence au terme «maudit » qui avait causé sa perte. Le système politique ottoman reposait alors sur un pouvoir central et deux principaux contrepouvoirs : les notables provinciaux (ayan) et le corps des janissaires (yeniçeri oca $\breve{g} l$ ). Les ayan devaient leur puissance à un capital politique, social et économique local, notamment à travers l'agriculture et les fermes fiscales. Le corps des janissaires, créé dès le XIV siècle comme une milice d'esclaves, dans la logique de l'idéal ottoman des administrateurs et militaires issus du devşirme (levée d'enfants chrétiens des territoires conquis) s'était « embourgeoisé », s'intégrant de plus en plus dans la société et l'économie urbaines, ce qui les rendait encore plus redoutables aux yeux du palais.

Mahmud II signera en 1808 le «pacte d'alliance» (Sened-i Ittifak) avec les principaux ayan, reconnaissant par là leur statut en échange de leur soutien. Néanmoins, quelques années plus tard, il s'engagera dans une politique visant à réduire l'autorité et l'autonomie de ces magnats des provinces. Toutefois, ce n'est que bien plus tard qu'il osera enfin s'attaquer aux janissaires, cette fois-ci de manière décisive. L' « heureux événement » (Vak'a-i Hayriyye) du 16 juin 1826 anéantira manu militari le corps des janissaires, bannissant jusqu'à la mémoire de ce corps et de ceux qui lui étaient affiliés. 
La destruction des janissaires eut lieu en plein milieu du long conflit qui opposa l'État aux insurgés grecs, de 1821 à la constitution d'un État grec indépendant en 1830. Ce qui avait commencé comme une simple rébellion s'était rapidement teinté d'idéologie et de politique internationale. Si les puissances européennes se tinrent d'abord à l'écart de la question, l'opinion publique en Europe, motivée par le philhellénisme, prit rapidement la défense de la cause grecque. Les massacres de l'île de Chio (1822) et la chute de Missolonghi (1826) ne firent qu'accrô̂tre ce phénomène, exacerbé par la présence de nombreux volontaires européens venus défendre, l'arme au poing, une Grèce de plus en plus idéalisée.

Pour les Ottomans, c'est la découverte d'une solidarité entre l'Europe et les insurgés grecs dont les fondements idéologiques et sentimentaux paraissent surprenants. La documentation concernant la citadelle d'Athènes - l'Acropole pendant le siège de la ville par les troupes ottomanes en 1826 illustre particulièrement bien la manière dont les Ottomans sont amenés à découvrir l'importance d'une idéologie européenne qui ne leur était guère familière. Pressés par la diplomatie européenne à épargner les monuments antiques auxquels eux-mêmes n'attachaient guère d'importance, les Ottomans sont forcés de constater que ces « vieilles pierres » ont une valeur symbolique qui dénote un changement radical dans la manière dont la politique et la diplomatie se font désormais dans ce nouvel ordre du monde.

Constatation qui permet une transition vers les premiers cours de l'année 20182019, qui débuteront avec les chamboulements internes des années 1820 et 1830, de la destruction des janissaires à la crise égyptienne, et dont le décret des Tanzimat (1839) constituera une sorte de dénouement. Cependant, il s'agit aussi d'une transition vers le thème central du colloque de cette année, qui portera sur l'histoire de l'archéologie et, notamment, la «découverte» de la Phénicie dans la seconde moitié du XIX ${ }^{\mathrm{e}}$ siècle.

\section{COLLOQUe - LA DÉCOUVERTE DE LA PHÉNICIE AU XIX` SIÈCLE : SIDON ENTRE LA FRANCE, L'EMPIRE OTTOMAN ET LE LIBAN}

Pour sa première journée d'études, la chaire internationale d'histoire turque et ottomane a choisi de traiter d'un sujet qui recoupe plusieurs intérêts et disciplines : la découverte archéologique de la Phénicie au XIX ${ }^{\mathrm{e}}$ siècle, avec un accent particulier sur le site de Sidon, aujourd'hui la ville libanaise de Saïda, à une quarantaine de kilomètres au sud de Beyrouth. Confluence tout d'abord entre l'archéologie proprement dite et l'histoire ; celle de l'archéologie et des collections, bien sûr, mais aussi celle de l'Empire ottoman et plus particulièrement du Liban, au cœur de ce que l'on a appelé la «Question d'Orient». Il en découle une forme de rivalité entre la France, acquérant dans la région une influence croissante qui culminera avec un mandat après la Grande Guerre, et l'Empire ottoman, tentant de maintenir son autorité dans une province à la démographie complexe, de plus en plus fortement imbriquée dans un contexte quasi colonial. Enfin, une histoire plus ciblée sur des hommes, acteurs principaux de cette «découverte»: Ernest Renan, historien, philologue et professeur au Collège de France, dont la mission de Phénicie (18601861) jouera un rôle pionnier dans la foulée des premières découvertes à Sidon ; mais aussi Osman Hamdi Bey, dont les fouilles de 1887 mettront au jour une riche nécropole dont les sarcophages, défrayant la chronique, lui fourniront les moyens de propulser l'archéologie et la muséologie ottomanes au-devant de la scène 
internationale. Il s'agit aussi d'acteurs secondaires, dont le rôle est mineur, souvent ambigu, tels les membres de la famille Durighello, chasseurs d'antiquités selon certains, et des notabilités de la région, tels l'ingénieur Béchara Deb ou les dynasties locales des Jumblatt et des Arslan. De fouille en fouille, de sarcophage en sarcophage, des spécialistes de ce vaste et complexe sujet dévoilent et examinent les péripéties de cette fascinante histoire qui nous mène jusqu'à la question de la gestion et la perception de ce patrimoine de l'effondrement colonial au présent.

\section{RECHERCHE}

Une partie considérable de mes recherches pendant l'année académique 20172018 a porté sur l'histoire de l'archéologie dans l'Empire ottoman, objet principal de mes recherches depuis quelque temps. Dans ce cadre, j'ai notamment pu achever le classement des archives historiques du Musée archéologique d'Istanbul, anciennement Musée impérial, en attente depuis des décennies du fait de la barrière linguistique : la presque totalité des fonds conservés sont en effet en français. Or il s'agit là d'une documentation d'une importance cruciale pour une compréhension $\mathrm{du}$ fonctionnement interne de l'institution et de ses rapports avec le monde archéologique occidental. La fin du classement, qui sera suivie dans le courant de l'année 2018-2019 par l'élaboration et, éventuellement, la publication d'un inventaire raisonné, devrait déboucher sur l'ouverture des archives à la recherche, tant attendue par nombre de chercheurs désirant se pencher sur la dimension locale de l'archéologie et la muséologie ottomanes.

Évidemment, s'il s'agit là d'un «service public » rendu à la communauté des chercheurs et au musée lui-même, ce travail de classement fait aussi partie de l'élargissement et de l'approfondissement de mes propres recherches sur le sujet. L'année académique a été particulièrement fructueuse en matière de projets menés à bien dans ce but, puisque j'ai pu publier trois articles de fond sur des questions touchant à la période la plus méconnue du Musée impérial, soit les trois décennies qui suivirent sa fondation en 1846. Ce travail critique a été mené à partir d'une documentation externe au musée, celle des archives de l'État, puisque à cette époque le musée - et c'est l'un des arguments de base de mes trois contributions - n'avait pas encore acquis les moyens et les qualités d'une véritable institution. La mise au jour des archives du musée me permettront désormais de me pencher systématiquement sur la période plus «mûre» de l'établissement, mais avec un accent particulier sur les dynamiques locales de la discipline. Un premier projet dans ce sens portera vraisemblablement sur le petit monde des archéologues et épigraphistes amateurs grecs des provinces. C'est là un sujet qui a été de tout temps ignoré par le «grand» récit occidental de l'archéologie, mais surtout par l'histoire turque de la discipline, toujours réfractaire à la reconnaissance de la contribution d'acteurs non musulmans et non turcs.

Deuxième volet de mon programme de recherche pour l'année, j'entame un programme de publication intégrale et commentée des écrits personnels du prince Salahaddin Efendi (1861-1915), fils du sultan Murad V, au règne si éphémère, de juin à août 1876. Il s'agit là d'une documentation exceptionnelle par la quantité, la variété et, bien sûr, la qualité. Assigné à résidence dans un palais coupé du monde avec son père et tout leur entourage pendant les vingt-huit ans qui suivirent la déchéance de Murad, jusqu'à sa mort en 1904, le jeune prince avait tout le temps et 
toutes les raisons de s'adonner à un exercice personnel, mémoriel et sentimental rarement pratiqué par ses contemporains, y compris les membres de l'élite culturelle et politique de l'Empire. Au demeurant, plus que le statut princier de son auteur, ce qui rend cette documentation exceptionnelle, c'est tout au contraire son profil tout à fait ordinaire d'un point de vue culturel et intellectuel. Dépourvu d'une véritable éducation, mais nourri d'une connaissance superficielle du monde qui l'entoure, Salahaddin Efendi se fait l'interprète des mentalités, des curiosités, des inquiétudes de l' «Ottoman moyen », autant de visions et de phénomènes qui nous échappent du fait du silence documentaire qui caractérise cette catégorie d'individus.

Il s'agit donc d'un projet à double emploi : d'un côté, la publication intégrale d'un texte dont la richesse et la complexité nourriront la discipline d'un matériau d'une originalité et d'une diversité remarquables, tandis que, de mon point de vue, s'annonce le début d'une exploitation plus ciblée de certains aspects particuliers du corpus ainsi constitué. Une première tentative a débouché sur un article portant sur les « voix » féminines que cette documentation essentiellement masculine n'arrive pas totalement à taire et qui deviennent d'autant plus importantes que l'univers quasi carcéral dans lequel évolue le jeune prince est presque exclusivement féminin et servile. L'article en question paraîtra dans le numéro de la revue Clio $(42,2018)$ consacré au thème de «Femmes, genre, histoire ».

\section{PuBLiCATIONS}

Alloul H., Eldem E. et Smaele H. de (dir.), To Kill a Sultan. A Transnational History of the Attempt on Abdülhamid II (1905), Londres, Palgrave Macmillan, 2018, DOI : 10.1057/978-1137-48932-6.

ELDEM E., L'Empire ottoman et la Turquie face à l'Occident, Paris, Collège de France/Fayard, coll. «Leçons inaugurales du Collège de France », $\mathrm{n}^{\circ}$ 275, 2018 ; édition électronique : Collège de France, https://books.openedition.org/cdf/6192, DOI : 10.4000/books.cdf.6192.

ELDEM E., «Early Ottoman archaeology : Rediscovering the finds of Ascalon (Ashkelon) : 1847 », Bulletin of the American Schools of Oriental Research, $\mathrm{n}^{\circ}$ 378, 2017, p. 25-53, DOI : 10.5615/bullamerschoorie.378.0025.

ELDEM E., «Of bricks and tiles: The history of a local industry in the area of Mürefte : Thrace », in E. AKÇETIN et S. FAROQHI (dir.), Living the Good Life : Consumption in the Qing and Ottoman Empires of the Eighteenth Century, Leyde/Boston, Brill, 2017, p. 433-473, DOI : 10.1163/9789004353459_019.

ELDEM E., "The search for an Ottoman vernacular photography », in M. RITTER et S.G. SCHEIWILLER (dir.), The Indigenous Lens? Early Photography in the Near and Middle East, Berlin, De Gruyter, coll. «Studies in Theory and History of Photography : Schriften der Lehrund Forschungsstelle für Theorie und Geschichte der Fotografie am Kunsthistorischen Institut der Universität Zürich », nº 8, 2017, p. 29-56, DOI : 10.1515/9783110590876-004.

Alloul H., Eldem E. et Smaele H. de, «Introduction: Anatomy of the Yildiz bombing: Tracing the global in the particular », in H. Alloul, E. Eldem et H. de SMAELE (dir.), To Kill a Sultan. A Transnational History of the Attempt on Abdülhamid II (1905), Londres, Palgrave Macmillan, 2018, p. 1-33, DOI : 10.1057/978-1-137-48932-6_1.

Eldem E., «Epilogue », in H. Alloul, E. Eldem et H. de Smaele (dir.), To Kill a Sultan. A Transnational History of the Attempt on Abdülhamid II (1905), Londres, Palgrave Macmillan, 2018, p. 265-271, DOI : 10.1057/978-1-137-48932-6. 
ELDEM E., «The (still)birth of the Ottoman "Museum": A critical reassessment », in M. Wellington Gahtan, et E.-M. Troelenberg (dir.), Collecting and Empires. An Historical and Global Perspective, Turnhout, Brepols, 2018, p. 258-285.

ELDEM E., «Et si Diane n'était pas lesbienne ? Biographie critique d'une statue(tte) d'Artémis du Musée archéologique d'Istanbul », Bulletin de correspondance hellénique, vol. 141, n 1 , 2017, p. 389-446.

ELDEM E., «A French view of the Ottoman-Turkish wine market, 1890-1925 », in L. THYSŞENOCAK (dir.), Of Vines and Wines. The Production and Consumption of Wine in Anatolian Civilizations through the Ages, Louvain/Paris/Bristol, Peeters, coll. " Ancient Near Eastern studies : Supplement », no 51, 2017, p. 169-209.

ELDEM E., «Turcos y árabes en la Alhambra : el descubrimiento otomano de al-Ándalus », in J.A. GonZÁleZ AlCANTUD (dir.), Leones y doncellas : dos patios palaciegos andaluces en diálogo cultural (siglos XIV al XXI), Grenade, Universidad de Granada, 2018, p. 317-338.

ELDEM E., «The Ottomans and the Alhambra: Inspiration or ignorance? », in F. GIESE et A. VArela Braga (dir.), The Power of Symbols. The Alhambra in a Global Perspective, Berne, Peter Lang, 2018, p. 97-110, DOI : 10.3726/b10940.

ELDEM E., «Cultural networks, public knowledge : Introduction », in A. DALACHANĒs et V. LEMIRE (dir.), Ordinary Jerusalem 1840-1940. Opening New Archives, Revisiting a Global City, Leyde/Boston, Brill, 2018, p. 283-286, DOI : 10.1163/9789004375741_018.

ELDEM E., «Ottomans at the Alhambra, 1844-1914: An Investigation into the perception of Al-Andalus by Ottoman subjects in times of modernity », Turcica, vol. 49, 2018, p. 239-359, DOI : 10.2143/TURC.49.0.3285083. 
